\title{
L'Énergie électrique dans l'État de Săo-Paulo
}

\section{Electric power in the State of Sào Paulo}

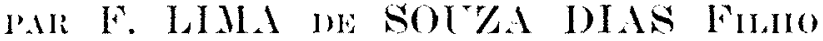

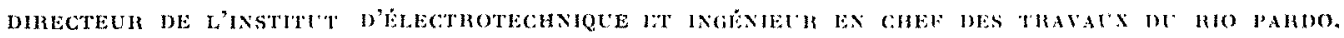 \\ ET .J. (XLLALIO) DA ROCHA \\ assistane
}

Mistorique de la distribution de l'energie électrique dans le são Panlo. Groupe de producteurs. Origines, emplacements, puissances et développements des deux grands producteurs at des groupes indépendants. Insu/fisances constatèes et projets d'extension. Intervention de letat; son programme. Caractéristiques des centrales en projet.

\begin{abstract}
Account of the distribution of electric energy in Srio Paulo. Grouping of producers. Origins, inslallations, poner and developments of the two largest producers and of the independent gronps. Inadequacios observed and extension projects. Intervention of the state; its programme. Charateristics of the proposed powet slations.
\end{abstract}

\section{I. - HISTORIQUE}

a) L'ENLRGIE A L'INTÉRIEUR DE L'EtTAT.

A la fin du siècle dernier, marqué par les progrès réalisés dans lelal de São Paulo (p)ogres dus principalement à la cullure du cafó), apparurent les premicres entreprises industrielles d'énergie électrique.

Elles se signalaient par leur caractere presque exclusivement municipal, l'installation des centrales hydro-électriques étant due à l'initiative des planteurs de café ou des détenteurs de capilaux qui s'unissaient dans le cadre d'une ville pour organiser de pelites entreprises parliculicres productrices d'énergie, obtenant des concessions municipales pour leur exploitation.
Les motifs qui déterminerent l'apparition de ces petites entreprises Jocales ont dé, avant toul, les distances relativement grandes entre les cenlres de consommation alors on formation, lichelle relativement réduile de leurs besoins et, entre autres, la mentalité * coloniale * des habilanls de l'intérieur du pays qui, poussés par des nécessilés geographiques et politiques, vivaient en autarchie.

C'est ainsi que, jusqu'en 1930, les villes de l'intérieur ont va prospérer des dizaines de pelites entreprises comprenant des installations hydro-cilectriques peu puissantes, dont la majeure partie est encore en service, bien que dans un état d'épuisemenl presque complet. 


\section{b) L'ENIRGG baNS LA CAPITALF DF L'ETAT.}

Parallèlement à l'installation des petites entreprises a l'intérieur du pays, le début $d u x^{0}$ siècle a vu se créer, à São Paulo, sous l'impulsion d'Alexandre Mackenzie, une entreprise plus importante qui a su intéresser aux installations hydro-électriques de São Paulo, des capilaux, canadiens pour la plupart. Ce groupe a su prévoir l'avenir industriel de la ville de São Paulo, centre naturel vers lequel convergent toute la richesse et toute la production de l'arrière-pays et halte obligatoire sur la route du port de Santos, seuil el capitale du pays.

\section{c) Fonmation des groupes actuels.}

Après la grande crise économique de 1929, l'Etat a connu, en 1930, une longue période de stagnation et mème de régression, à tel point qu'en de nombreuses régions, et principalement dans la région appelée «vieille zone», région de la Cie des Chemins de Fer de Mogiana, on a noté une sérieuse diminution de consommation de l'énergie électrique; cet état de choses a placé la plus grande partie des petites entreprises dans une situation telle, qu'elles se sont désintéressées de leurs propres installations, devenues peu rentables.

Une telle situation a facilité la formation du groupe de Ia Cia Paulista de Força e Luz (du groupe américain Bond and Share) qui, avec des capitaux américains (le cours du dollar étant très haut par rapport au cruzeiro), a, en offrant des prix intéressants, acheté un grand nombre de petites entreprises de l'intérieur, contrôlant presque ainsi les deux tiers de cet Etat.

Dans les régions de Alta Sorocabana et de Alta Paulista, régions de formation très récente, florissaient quelques compagnies indépendantes, qui, pourtant, du fait de leur caractère régional, ne pouvaient se développer de façon à soutenir le rythme du progrès et des nécessités de la résion, surtout en tenant compte de la loi dite du « coùt historique » relative à la rémunération du capital (loi de 1934), loi dont les effets malheureux se firent sentir d'une façon accenluée après la grande inflation monétaire brésilienne, à partir de 1939.

De tout ceci, il résulte que l'exploitation de l'énergic électrique de l'Etat de São Paulo est actuellement dirigée par les quatre grands groupements suivants :

a) Light \& Power (capitaux canadiens), desservant São Paulo, la capitale, ainsi que Santos, Jundiai et les villes de la vallée du Paraiba entre São Paulo et la capilale fédérale, soit au total 3.293.000 habitants;

b) Cia Paulista de Força e Luz (capitaux américains) dont le réseau couvre près des deux tiers

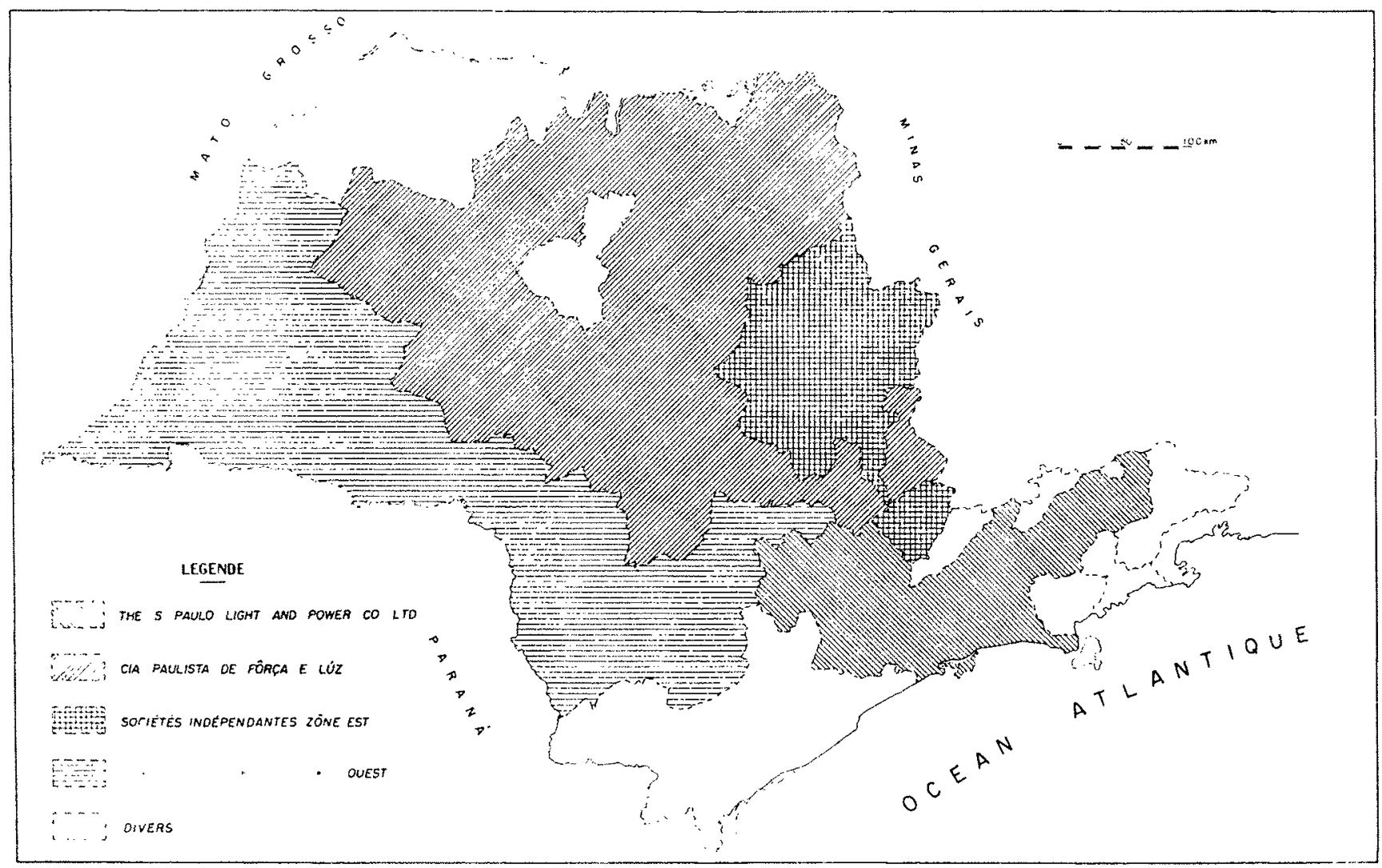

Fiki, 1, ...- Etal de Sĩo Paulo. 
TABLEAU STATISTIQUE POUR L'ANNEE 1950, DANS L'ETAT DE SAO-PAILO

\begin{tabular}{|c|c|c|c|c|c|}
\hline CompatiNies & Poptiation & $\begin{array}{l}\text { Puissance } \\
\text { installée }\end{array}$ & $\begin{array}{l}\text { Puissance } \\
\text { par tête } \\
\text { (watts) }\end{array}$ & $\begin{array}{l}\text { Pronurcriox } \\
\text { annuelle } \\
\text { (kWh) }\end{array}$ & $\begin{array}{l}\text { Propercron } \\
\text { partetele } \\
\text { (kWh) }\end{array}$ \\
\hline ThE SAO Patlo Light \& Power. . . . . & 3.293 .445 & 622.974 & 189 & 3.349 .335 .998 & 1.117 \\
\hline \multicolumn{6}{|l|}{ EsT : } \\
\hline S/A Central El. R. Claro.......... & 227.905 & 13.783 & 60 & 57.675 .740 & 253 \\
\hline Cia. Prada Eletricidade .......... & 41.454 & 1.986 & 48 & 5.634 .542 & 136 \\
\hline Cia. Paul. de Eletricidade . . . . . . . . . & 67.460 & 4.266 & 63 & 21.006 .672 & 311 \\
\hline Cia. Eletric. S. Simāo-Cajurú. . . . . . . . & 49.005 & 1.618 & 33 & 8.450 .000 & 172 \\
\hline Cia. Sanjoan. Eletricidade......... & 60.003 & 2.935 & 49 & 15.709 .110 & 262 \\
\hline Cia. Paul. En. Elétrica $\ldots \ldots \ldots \ldots \ldots$ & 67.351 & 3.979 & 59 & 12.540000 & 186 \\
\hline Empresa Elétr. Bragantina......... & 92.248 & 3.310 & 36 & 13.961 .338 & 151 \\
\hline Cia. Força Luz Mocóca. ....... & 31.760 & 1.188 & 37 & 8.006 .640 & 252 \\
\hline \multicolumn{6}{|l|}{ Ouest : } \\
\hline Cia. Força Luz Sta Cruz $\ldots \ldots \ldots \ldots$ & 177.390 & 8.922 & 50 & 43.739 .735 & 247 \\
\hline Cia. Hidro El Paranapanema. . . . . . & $101.30 \overline{5}$ & 6.803 & 67 & 21.682 .000 & 214 \\
\hline Empr. El. Vale Paranapanema........ & 234.811 & 1.765 & 75 & 4.895 .547 & 21 \\
\hline Cia. Elétrica Caiuá. $\ldots \ldots \ldots \ldots \ldots \ldots$ & 396.296 & 4.928 & 12 & 17.743 .929 & \multirow{2}{*}{45} \\
\hline \multicolumn{5}{|l|}{ Drvens: } & \\
\hline Cia. Nac. En. Elétrica. ........... & 112.910 & 4.597 & 41 & 13.410 .063 & 119 \\
\hline Cia. Taubaté Industrial.......... & 28.627 & 2.435 & 85 & $9.538 .5(14$ & $33: 3$ \\
\hline Empr. Eletrica Sul Paulista..... & 52.320 & 2.052 & 39 & 6.286 .92 .3 & 120 \\
\hline Empr. Luz Força Tatuí-Tietê.... & 105.682 & 3.751 & 36 & 10.806 .972 & 102 \\
\hline Empr. Eletrica Piracaia.......... & 11.523 & 588 & 51 & 969.700 & 84 \\
\hline Cia. Sul Mineira Eletricidade..... & 13.201 & 235 & 18 & $368.28: 3$ & 28 \\
\hline Cia. Fiaç. Tecel. São Pedro........ & 6.419 & 1.655 & 258 & 5.562 .670 & 867 \\
\hline Cia. City de Santos (Docas) ........ & 251.737 & 14.710 & 59 & 69.027 .287 & 274 \\
\hline Empr. Hidro El. Jaguari. .......... & 7.025 & 1.368 & 189 & 3.500 .000 & 498 \\
\hline Empr. F. L. de Pederneiras. . . . . . . . . & - & -- & 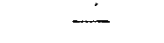 & - & - \\
\hline Cia. Independ. Eletric $\ldots \ldots \ldots \ldots \ldots \ldots$ & $\cdots$ & - & - & - & - \\
\hline \multirow[t]{2}{*}{ Empr. El. Paraibunense............ } & 17.093 & 80 & 5 & 263.940 & \multirow[t]{2}{*}{15} \\
\hline & 606.537 & 31.471 & & 119.734 .342 & \\
\hline
\end{tabular}

Fic. 2

de l'Etat, s'étendant sur de vastes régions et desservant 2.700.000 habitants;

c) Un groupe de sociétés indépendantes de petite envergure de la zone est, qui n'ont pas été absorbées par la Cia Paulista et qui dessert 800.000 habitants;

d) Un groupe de sociétés indépendantes de la zone sud-ouest de l'Etat, de formation plus récente, desservant 900.000 habitants;

e) Le reste est composé de quelques compagnies indépendantes, disséminées à travers l'Etat et qui ne s'incorporent pas d'une façon précise à tel ou tel des groupes précédents. Ces compagnies desservent environ 600.000) habitants.

La figure 1 est une carte de l'Etat de São Paulo montrant la répartition des quatre groupes cidessus.

La figure 2 constitue un tableat donnant, pour chacun des groupes et pour l'anné 1950, les puissances installées, la population et la consommation en $k$ Wh par habitant et par an. Enfin, sur une carte de l'Etat de São Paulo, sont figurées (fig. 3) toutes les centrales actuellement en service. 


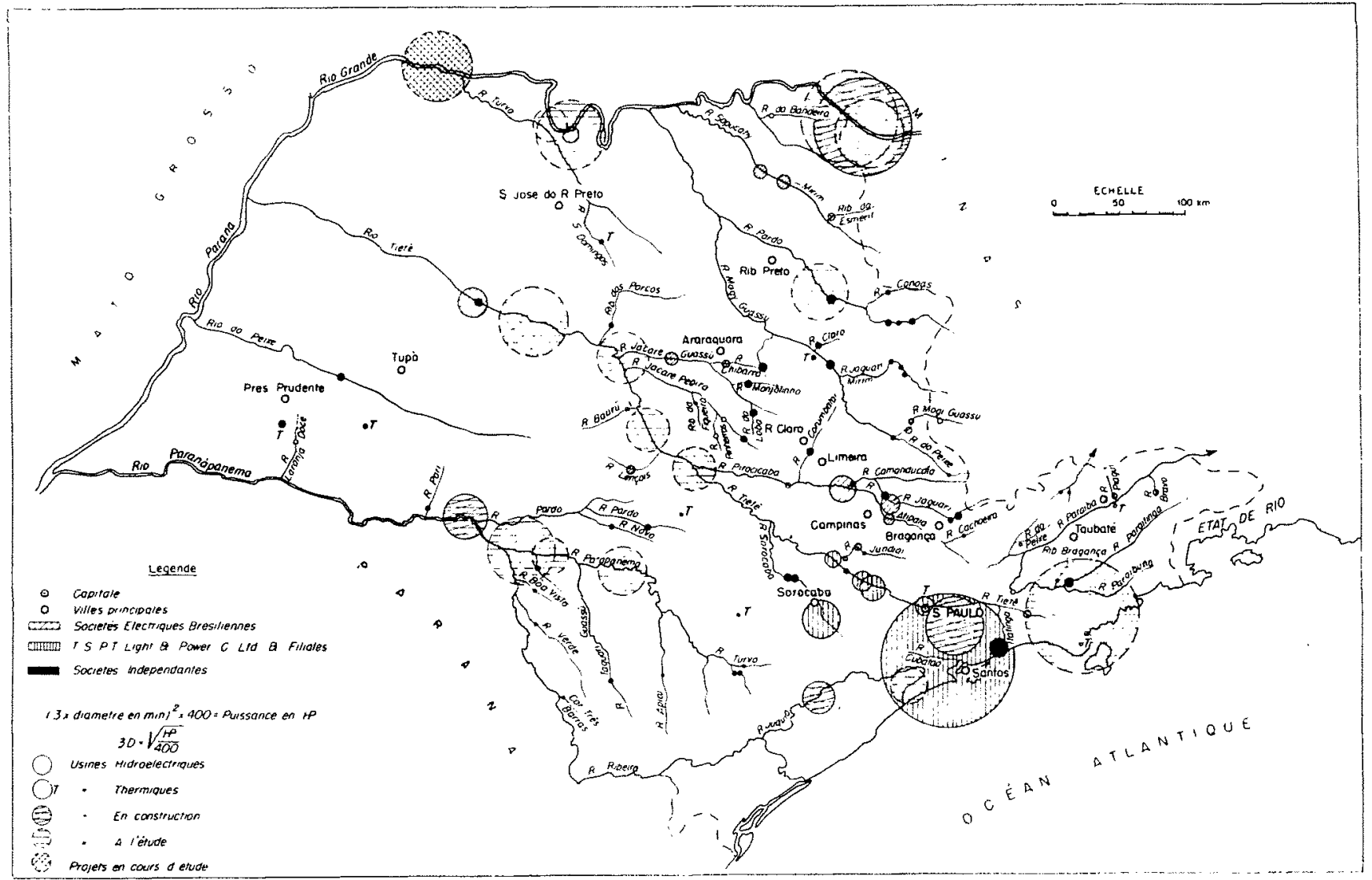

Fli, 3. -. Etat de São Paulo. Principales centrales électriques

\section{II. - LE DÉVELOPPEMENT DES GROUPEMENTS ET PROJETS ACTUELS}

\section{a) Light \& POWER.}

Le developpement du groupe Light \& Power est illustré clairement par les figures 4 (Courbe de progression du systène Light) et 5 (Evolution de la puissance installée et de la demande).

Dans les trente dernieres années, la charge a augmenté dans la proportion moyenne annuelle de $11,2 \%$. Si l'augmentation se poursuit à ce rythme, on devra prévoir, en 1961, une puissance de $1.100 .000 \mathrm{~kW}$.

Actuellement, ce groupement traverse certaines difficultés dans le domaine de la production d'énergie, difficultés qui apparaissent bien sur la figure 6 (Courbe de charge du systeme Light).

Les raisons de ces difficultés sont assez complexes et controversées; on en donne des interprétations très variées.

Pour faire face à cette situation et être à même de satisfaire les besoins à venir, la Light \& Power a dressé le programme des travaux suivants :
1. Centrale souterraine de Cubatĩo.

Puissance Prévision
en
MII

$$
\begin{aligned}
& \text { Groupe } N^{\circ} 9 \ldots .65 \quad 65 \\
& \ldots \quad N^{\circ} 10 \ldots .65 \quad 656 \\
& \text {...... } N^{*} 11 \ldots .65 \quad 1957 \\
& -\quad N^{\circ} 12 \ldots 6658
\end{aligned}
$$

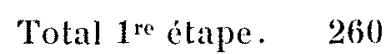

Groupe No $13 \ldots .656$

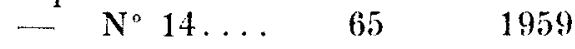

Total 2" étape. $\overline{130}$

Tolal des deux étapes 390

2. Centrale thermique de Piratininga.

$$
\begin{array}{crrr}
\text { Groupe } N^{\circ} 1 \ldots \ldots & 80 & 1954 \\
- & N^{\circ} 2 \ldots \ldots & 80 & 1954 \\
& & & \\
& & &
\end{array}
$$




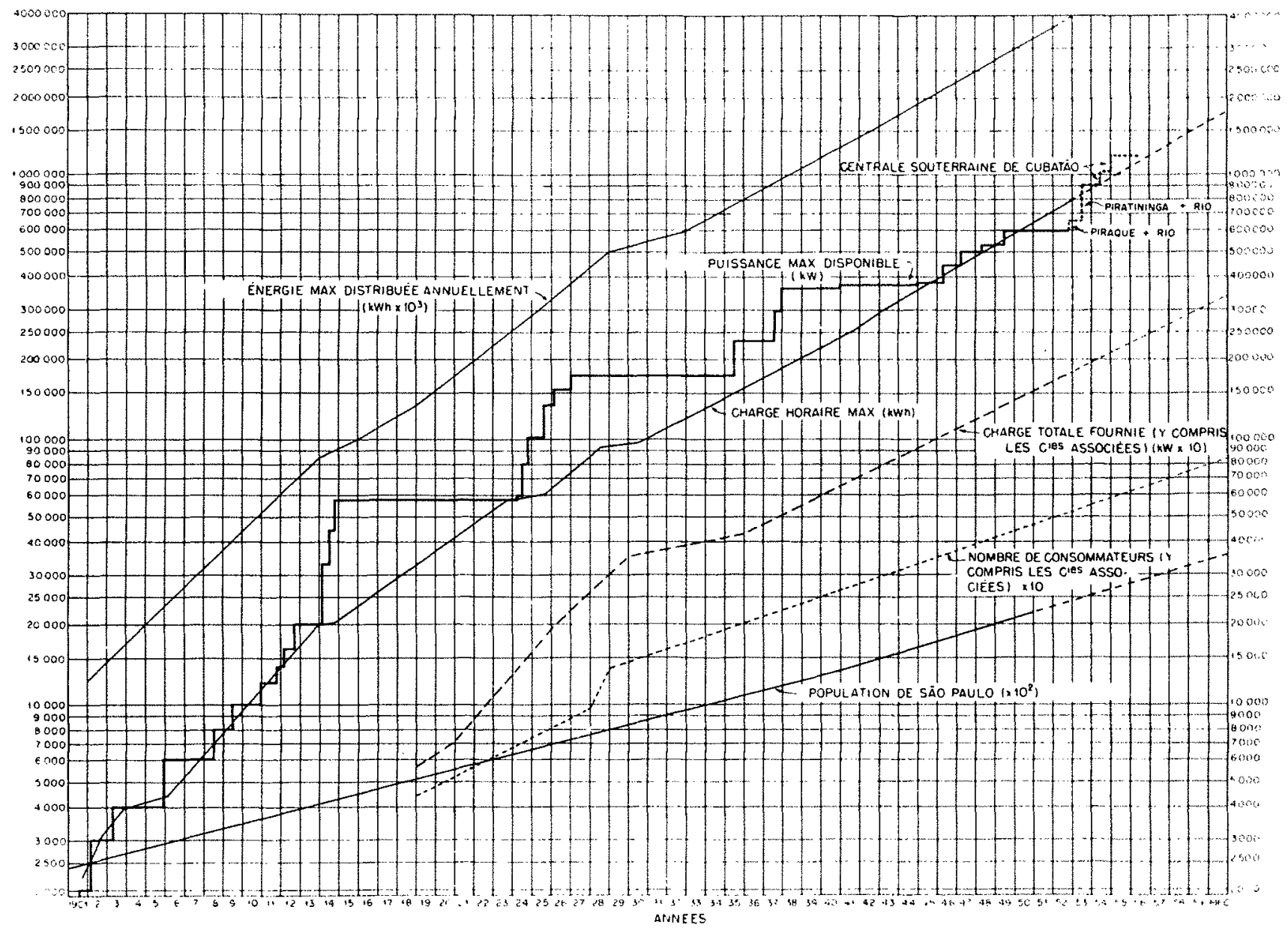

Fin. 4. -.- Courbe d'aceroissoment du systeme Lighl.

\section{Puissance pouvant ètre transportee} depuis le systeme de Rio (centrale de Forcacava) $\dot{a} S \tilde{a} o$ Paulo par la ligne de $220 \mathrm{kV}$ : limite actuelle............ $100 \mathrm{MW}$

Total général de la puissance à installer ................. $650 \mathrm{MW}$

juscu'en 1959 .

Afin de pouvoir installer dans la centrale souterraine de Cubatão les six groupes de $65 \mathrm{MIN}$ prévus, cette Société doit achever les travaux de dérivation de la totalité des eaux du Rio Tietè (même en période de crues) vers le réservoir "Billings», ce qui nécessite de mener à bien les travaux suivants :

\section{Canal de Pinheiros.}

a) Creusement des canaux Jaguaré, Rebouças et Dragagens;

b) Installation des pompes 3 et 4 dans la centrale élévatrice de Pedreira.
5. Centrale «Edgar de Souza».

a) Elévation du harrage;

b) Installation de la pompe d'élivation N"1.

\section{Construction du barrege de Piraporta.}

Tous les travaux mentionnés ci-dessus (ilems t, 5 et 6) pourront être achevés en même temps que les installations relatives à la centrale sonterraine de Cubatio.

La figure 7 donne le plan et le profil schématique du systime hydro-électrigue de la Light \& Power.

\section{b) Cor Paglista de Forca re I.e\%}

C'est la plus importante filiale de la Cia Auxiliar de Enmpresas Elétricas Brasileiras, section brésilienne de la Electric Bond \& Share C".

Elle dessert 136 localites, dont 134 dans l'Etal de São Paulo el 2 dans l'Etat de Minas Gerais, avec une population totale de pris de 3.000 .000 d'habitants. Dans cette région, sont situées les villes les plus récentes et les plus aptes à se 


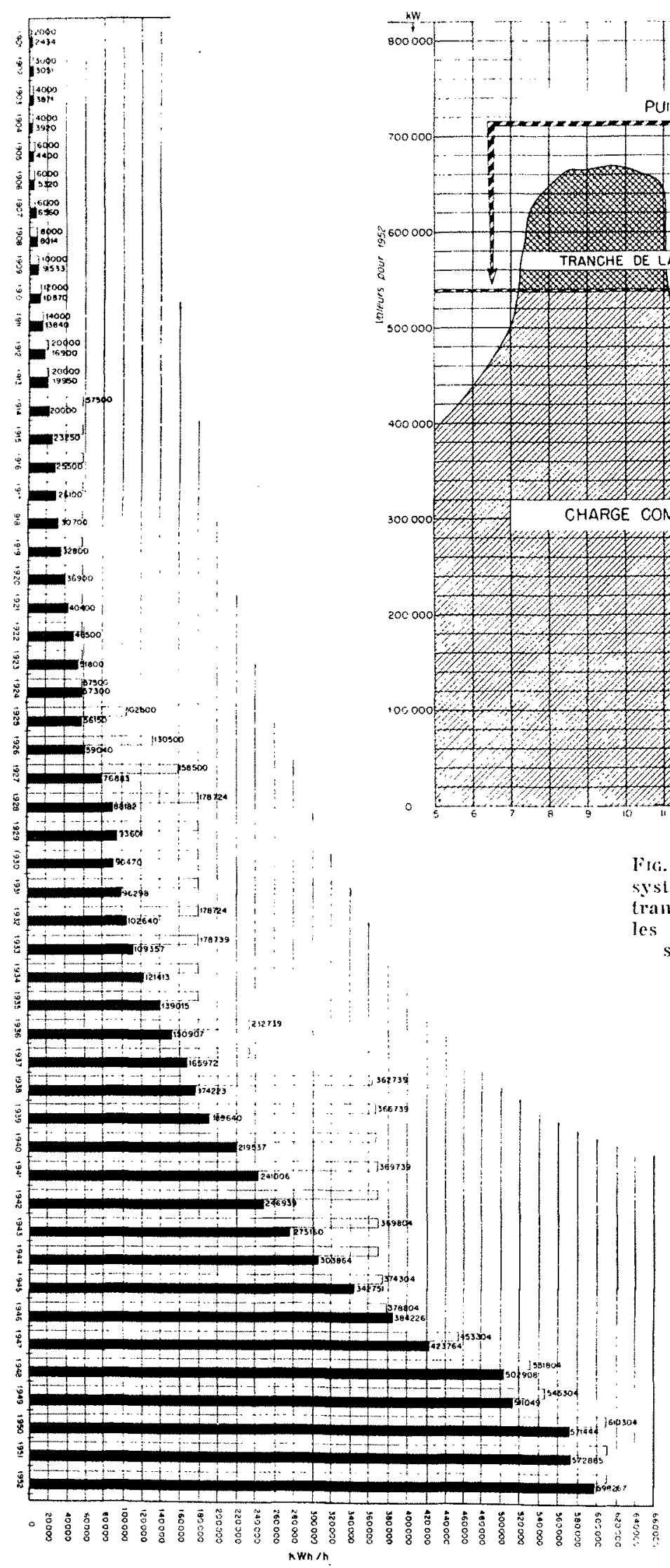

Fig. 5. - São Paulo Light and Power Co Ltd. Graphique donnant les pointes annuelles depuis $1901, y$ compris pompes et distributeurs.

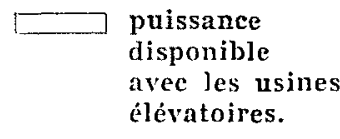

pointes.

La puissance disponible indiquée suppose toutes les centrales du réseau à leur puissance maximum, ce qui ne se produit qu’à la saison des pluies. En dehors de cette saison, la puissance indiquée comporte aussi celle des réserves hydrauliques qui n'est exploitée qu'exceptionnellement. 


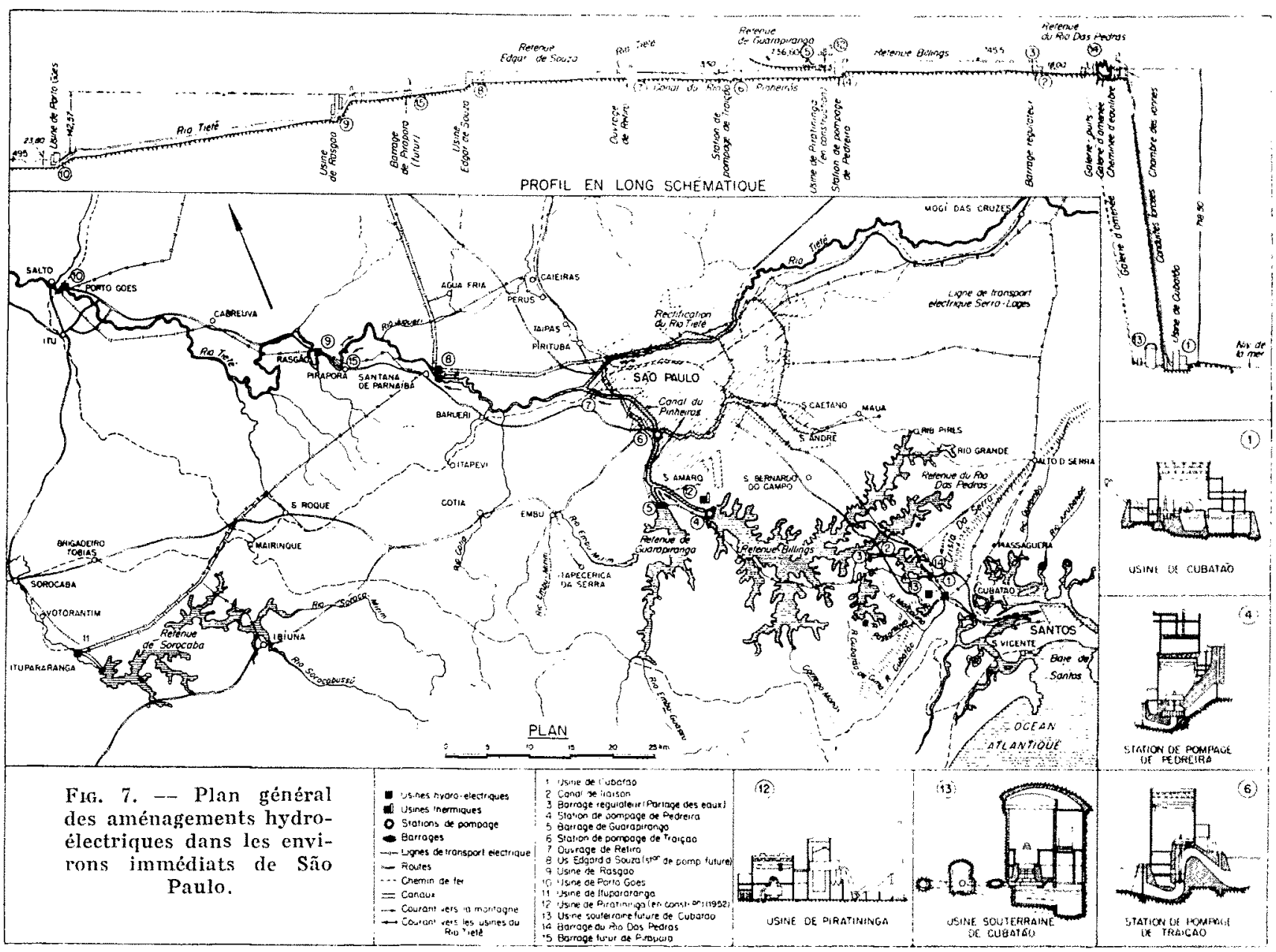

développer de l'Etal de São Paulo; nous mentionnerons spécialement les villes de Baurù, Ribeirão Preto, Campinas et São José do Rio Preto.

L'équipement de cette Société - en production, transport et distribution -- est le suivant :

- Centrales hydro-électriques... 17

- Puissance de production.....

- Lignes de transport et de dis-

$101.248 \mathrm{~kW}$ tribution ...............

- Postes abaisseurs...........

- Transformateurs de distribu-

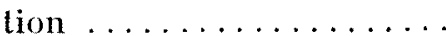

$9.155 \mathrm{~km}$ 101

La majeure partie des installations hydroélectriques est constituée par de pelites centrales, achetées au cours de 1930 et construites entre 1900 et 1928 .

Les deux installations principales sont :

- Centrale de Americana, sur le Rio Atibaia : $20.000 \mathrm{~kW}$

-- Centrale de Avanhandava, sur le Rio Tieté: $30.000 \mathrm{~kW}$,

construites respectivement en 1949 et 1945.

Le développement de la Cia Paulista de Força e Luz, dans la période 1942-1952, apparait sur la figure 8 , ou sont mises en lumiere les augmentations caractérisant le nombre des consommateurs, la valcur maximum de la charge horaire, el la production brute d'energie.

le manclue d'énergie dans la région desservie par celle Compangie est alarmant; il provoque une séricuse erise industrielle et suscite l'inquiélude de la population. Dans certaines localités, les coupures alteignent 10 heures par jour.

Pour salisfaire la demande sims cesse croissante, cette Socicti se propose de réaliser III plan quinquennal dont les éléments principaux sont les suivants :

1. Construction de la centrale thernique de Carioba, prévue pour mars $1954(30.000 \mathrm{~kW})$,

2. Augmentation de la puissance de la centrale hydro-électrique de Amerieana, oì un nouveau groupe entrera en service fin $1953(10.000 \mathrm{~kW})$,

3. Augmentation de la puissance de la centrale hydro-électrique de Jaguarí où un nou veau groupe entrera en service fin 1953 $(5.000 \mathrm{~kW})$, 


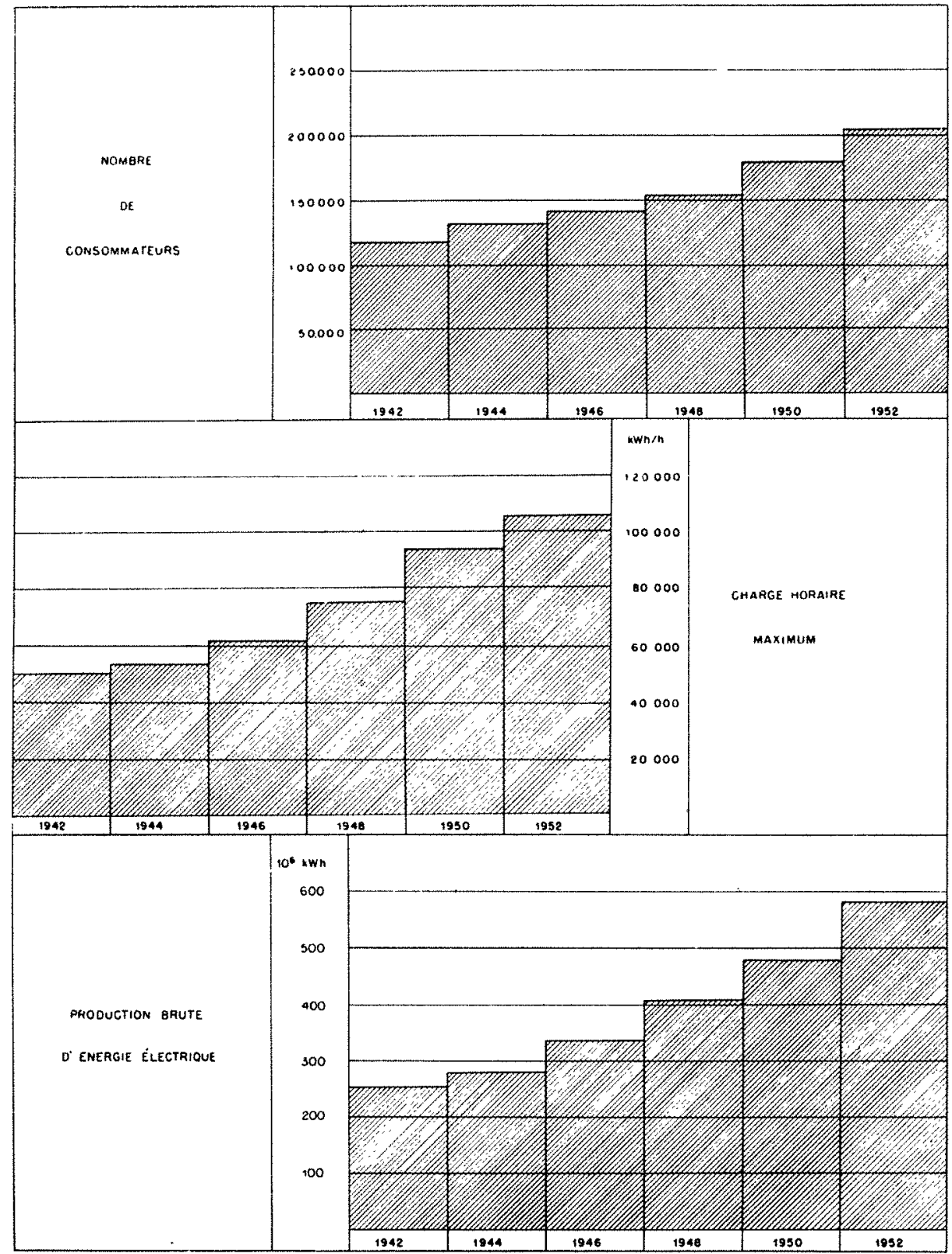

Fig. 8. - Courbes d'accroissement de la Cia Paulista de Fôrça e Luz.
4. Construction de la centrale hydro-électrique de Peixotos : installation des deux premiers groupes prévus pour entrer en service en $1956(80.000 \mathrm{~kW})$,

soit une augmentation totale de $125.000 \mathrm{~kW}$.

De toutes les installations prévues par le plan quinquennal, la centrale de Peixotos est la plus importante.

Située sur le Rio Grande, près de la frontière séparant les Etats de Minas Gerais et de São Paulo, cette centrale est prévue pour une puissance globale de $400.000 \mathrm{~kW}$ répartis en 10 groupes de $40.00 \mathrm{~kW}$. Usine et barrage formeront un seul bloc. Le barrage aura une hauteur maximum de $68 \mathrm{~m}$ et une longueur de $500 \mathrm{~m}$ environ.
$400.000 \mathrm{~m}^{3}$ de béton armé seront nécessaires à sa construction.

Le barrage créera un immense réservoir s'étendant sur $110 \mathrm{~km}$ le long du Rio Grande, et capable d'emmagasiner $4.000 .000 .000 \mathrm{de}^{3}$ utiles.

La centrale de Peixotos est la première étape de l'utilisation rationnelle du potentiel hydroélectrique du Rio Grande.

Ainsi donc, faisant suite au barrage de Peixotos, la Cia Paulista pourra, dans un proche avenir, procéder à deux nouvelles installations qui, avec Peixotos, fourniront près de $1.100 .000 \mathrm{~kW}$. Le réservoir de Peixotos assurera directement la régularisation de ces deux dernic̀res installations.

En plus de ces installations, qui se situeront en aval de la future centrale de Peixotos, la Cia 


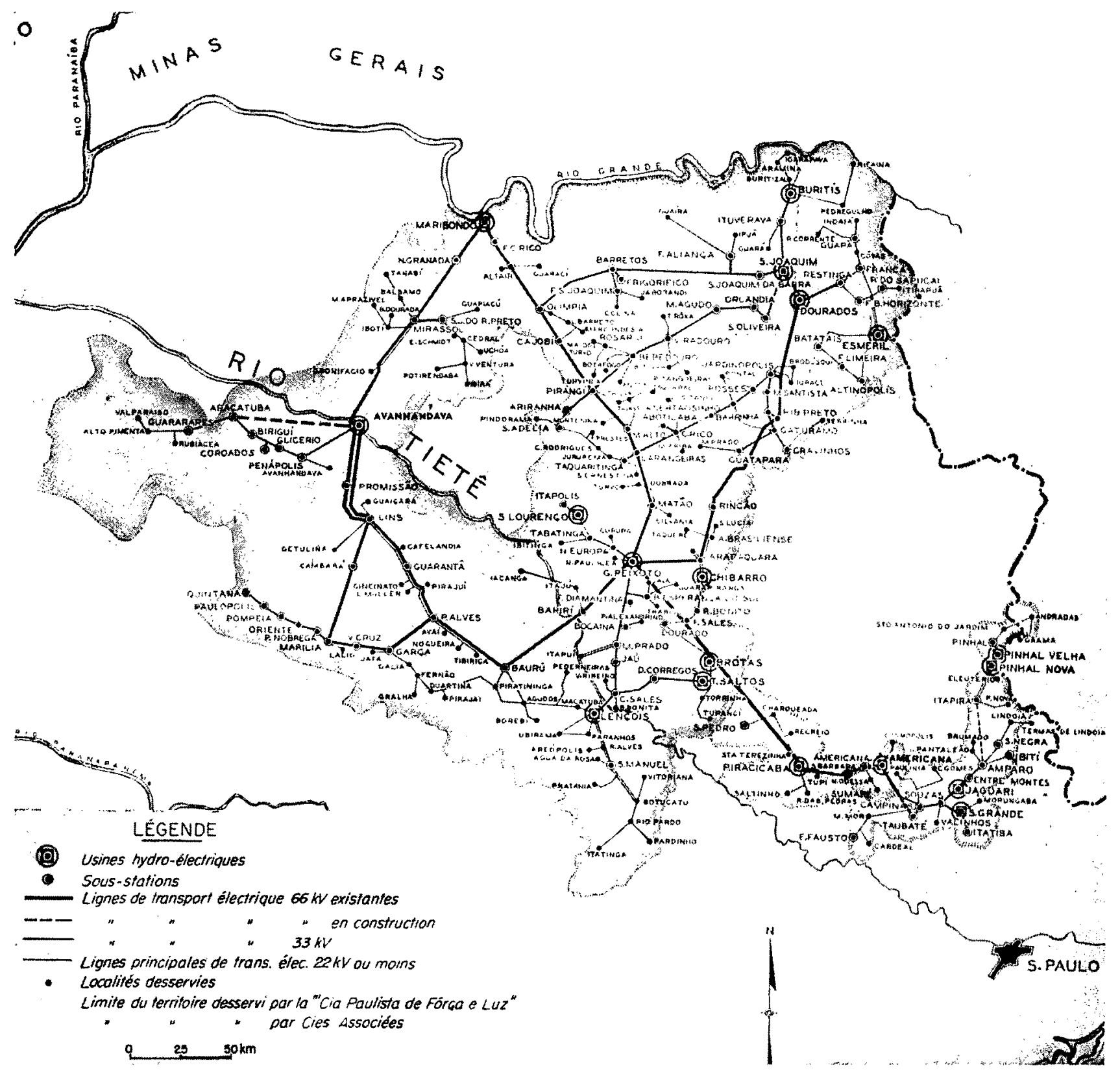

FIG. 9

Zone d'influence de la $C^{\prime n}$ Paulista

Paulista possède, à $200 \mathrm{~km}$ en aval, à la chute de Marimbondo, une pelite centrale qui, grâce à la régularisation dụ fleuve, pourra alteindre une puissance de $200.000 \mathrm{~kW}$.

Toutes ces installations sont à l'étude et leur programme est assez avancé; cependant, le point crucial réside dans l'aspect financier du problème, qui crée à la Cia Paulista de sérieuses difficultés.

En réalisant son plan quinquennal, la Cia Paulista se réservera les possibilités d'un développement encore plus grand dans un proche avenir.
En efrel, elle dessert dejà pres des deux tiers du teritoire de l'Etat el fournit de l'energie i lan région riche, bien desservie par des voies ferrés et possedant un bon réseau routier; la fourniture de l'énergie électrique suscitera dans celle vaste région un grand développement industriel et, compte tenu des besoins de décentralisation, l'industrie cherchera à se libérer de l'influence directe de la ville de São Paulo.

La figure 9 montre la zone d'influence de la Cia Paulista et le réseau ferroviaire de cette région. 


\section{(c) Grotplis indéplindayts.}

Nous désignons par le terme \& groupes indépendants $\gg$ les pelites sociétés productrices d'énergie électrique qui ont su éviter de se faire absorber par les grandes Compagnies.

Ces groupes peuvent être divisés en trois catégories géographiques, à savoir :

1. Groupe est;

2. Groupe sud-ouest;

3. Divers, répartis à travers le territoire de l'Etat.

Les compagnies faisant partie de ces groupes, toutes indépendantes, possèdent de petites installations hydro-électriques ou thermiques; elles n'ont pas pu suivre le développement de cette région, soil par suite de difficultés financières, soit, dans certains cas, par suite de l'inertie de leurs administrateurs; ceux-ci s'abstenant de les amplifier en raison, principalement, de la législation actuelle qui décourage tous les nouveaux investissements de capitaux dans les entreprises de production d'énergie électrique.

Le tableau figurant en figure 2 domne le ditail de ces groupes, des compagnies qui les composent, de leur puissance installée et de leur production annuelle.

Le tableau ci-dessous résume la situation :

\begin{tabular}{|c|c|c|c|c|c|}
\hline Groupes & $\begin{array}{c}\text { Popu- } \\
\text { lation }\end{array}$ & $\begin{array}{c}\text { Puis- } \\
\text { sance } \\
\text { ins- } \\
\text { tallée } \\
\mathrm{kW}\end{array}$ & $\begin{array}{c}\text { Watts } \\
\text { par } \\
\text { habit. }\end{array}$ & $\begin{array}{c}\text { Production } \\
\text { annuelle } \\
\text { kWh }\end{array}$ & $\begin{array}{c}\text { kWh } \\
\text { par an } \\
p^{r} \text { habit }\end{array}$ \\
\hline Zone Est.... & 637.186 & 33.065 & 52 & 142.984 .942 & 224 \\
Zone Ouest . & 909.802 & 22.418 & 24 & 88.061 .211 & 97 \\
Autres...... & 606.537 & 31.471 & 52 & 119.734 .342 & 197 \\
\hline Total...... & 2.153 .525 & 86.954 & 42,6 & 350.780 .495 & 172 \\
\hline
\end{tabular}

Une simple analyse comparative de ce groupe avec le groupe de Light \& Power nous permet de nous rendre compte de l'étal d'infériorité de celui-là.

Il convient de noter que si le taux moyen de consommation annuelle par habitant est de $172 \mathrm{kWh}$ pour l'ensemble de ce groupe, le même taux moyen est de 1.017 pour la Light \& Power. Si l'on compare d'autre part la puissance totale installée de ces divers groupes à celle de la
Light \& Power en fonction de la population, la différence est également flagrante.

Cet dat de complet déséquilibre est l'un des ficteurs du manque d'uniformité dans le développement de l'Elat et du dépeuplement de l'arrière-pays.

En vue de remédier à cette situation, le Gouvernement de l'Etat de São Paulo entreprend la construction de centrales hydro-électriques, conformément à un plan dont nous parlerons plus loin.

Il est intéressant de noter que la cause fondamentale de cet arrèt dans le développement des entreprises indépendantes réside dans le régime très désavantageux appliqué aux capitaux investis dans les installations productrices d'énergie, surtout si on les compare avec les avantages dont bénéficient les investissements immobiliers.

En ce qui concerne le programme propre de développement de groupes indépendants, il est passablement faible; ceci est dù, principalement, aux médiocres ressources financières de ces entreprises. Elles se sont bornées presque exclusivement à installer des groupes thermiques de secours destinès à atténuer le manque d’énergie qui, en période d'étiage, présente un caractère presque tragique: mesure précaire qui ne résoud nullement le problème.

Quelques compagnies appartenant au groupe indépendant ont entrepris de réaliser des installations hydro-électriques de faible envergure, à la mesure de leurs possibilités financières; toutes se sont heurtées à de sćricuses difficultés au cours des travaux.

La Cia Nacional de Energia Elétrica qui dessert la ville de Catanduva a achevé les études et les travaux de Génie Civil relatifs à l'installation de deux groupes de $4.600 \mathrm{~kW}$ chacun dans la centrale de Avanhandava, sur la rive droite du Tietê; mais l'achèvement de ce projet dépend des crédits obtenus pour l'achat du matériel hydro-électrique.

La Cia Paulista de Energia Elétrica desservant São-Carlos, a importé un équipement hydroélectrique de $5.000 \mathrm{~kW}$ destiné à sa centrale de Capão Preto qu'elle n'est pas en mesure d'achever, manque de fonds.

La centrale de Rio Claro a importé l'équipement hydro-électrique de $8.000 \mathrm{~kW}$ de l'usine de Jacutinga, sur le Rio Mogi-Guacú, mais elle se heurte à de sérieuses difficultés financieres pour entreprendre les travaux de Génie Civil.

D'autres compagnies ont réussi à mener à bien de petites installations hydro-électriques et it acheter des groupes Diesel de secours, parvenant ainsi à pallier aux besoins les plus urgents en énergie. 


\section{III. - PROGRAMME DE L'ÉTAT}

Etant donné les difficultés financières des compagnies indépendantes et comple tenu du programme insuffisant du groupe Light, une aide active de l'Etat dans le domaine de la production d'énergie électrique est devenue une nécessité pressante.

L'Elat a done pris la decision de construire quelques centrales hydro-électriques el a élaboré un plan général d'électrification dont les objectifs, dans leurs grandes lignes, sont les suivants :

a) Fournir de l'énergie aux compagnies independantes qui assureraient sa distribution;

b) Créer des disponibilités d'énergie à lintérieur de l'Etat, dans les zones défavorisćes, afin de couvrir Ie déficit actuel et le déficit prévú pour le système Light.

Ce programme a pour but de ereer une abondance d'énergic à l'intélieur de l'Etat, en opposition avec la carence qui se manifeste dans sa capitale - ce qui sera d'une srande portée économique et sociale - et de provoquer ainsi la décentralisation de l'industrie, qui est actuellement concentrée dans la ville de São Paulo, ce qui entraîne de graves conséquences sociales et politiques;

c) Le plan général de l'Etat est encore à l'étude; ses caractéristiques essentielles sont les suivantes : relivement économique général de l'Etat et études préliminaires générales de ses possibilités hydro-ilectriques, afin que lon puisse envisager l'interconnexion des divers syslimes el établir l'échelonnement des diverses réalisalions.

Avant mêne d'avoir achevé son plan général d'électrification, l'Etat a entrepris, devant la gravilé de la siluation créée par le manque d'énergie, la construction de quelques centrales hydroélectriques, destinées à fournir les groupes indépendants, à savoir :

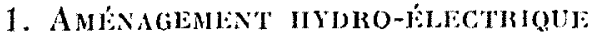 Di Paranapanema S.A.}

donl la majeure parlic apparlient a l'Elal, situé dans la zone sud-ouest de l'Etal el destini à alimenter les compagnies independantes el les
Chemins de Fer de Sorocabana, suivant le tableau ci-dessous :

\begin{tabular}{|c|c|}
\hline \multicolumn{2}{|c|}{$\begin{array}{c}\text { Siluation actuelle des entreprises } \\
\text { concessionnaires } \\
\text { dans la zone de Paranapanema }\end{array}$} \\
\hline COMPAGiNes & POPLLATION \\
\hline Ciat Elétrica Caituá........... & 385.100 \\
\hline $\begin{array}{l}\text { Empresa Elérica Vale do Parana- } \\
\text { panema } \ldots \ldots \ldots \ldots \ldots \ldots \ldots \ldots \ldots\end{array}$ & 353.000 \\
\hline Cia Luz e Forga Sta Cruz. . . . . & 217.200 \\
\hline 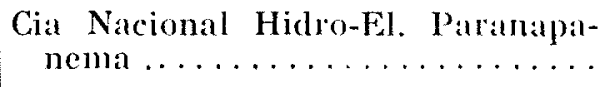 & 390.000 \\
\hline Empresat de Londrina S.A....... & 204.757 \\
\hline & 1.550 .0 .57 \\
\hline
\end{tabular}

La Centrale de Salto Grande est en construction et doil comporter :

- 4 groupes de $15.000 \mathrm{~kW}^{T}$, au lotal: $60.000 \mathrm{~kW}$.

Celle centrale a déja commandé ses 4 groupes el entrera en service a la fin de 1956 .

Dans le cadre de l'amenagement hydro-cleceIrique du Paramapanema, il a ble procede a l'étude des centrales suivanles:

(1) Juru-Mirim, qui commandera la régularisation annuelle du fleuve... 100.000 li 19

b) Pirajú $\ldots \ldots \ldots \ldots \ldots \ldots \ldots \ldots$

c) Itararé

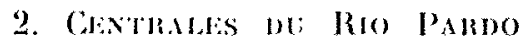

On éludie acluellement, avec possibilite do construction immédiate, les centrales de Limoeiro el de Euclides da Cunha, qui seront à la charge du Département des Filux el de l'Energic Electrique du Secretariat des Transports el des Travaux Publies de l'Elal de Sío Paulo. 


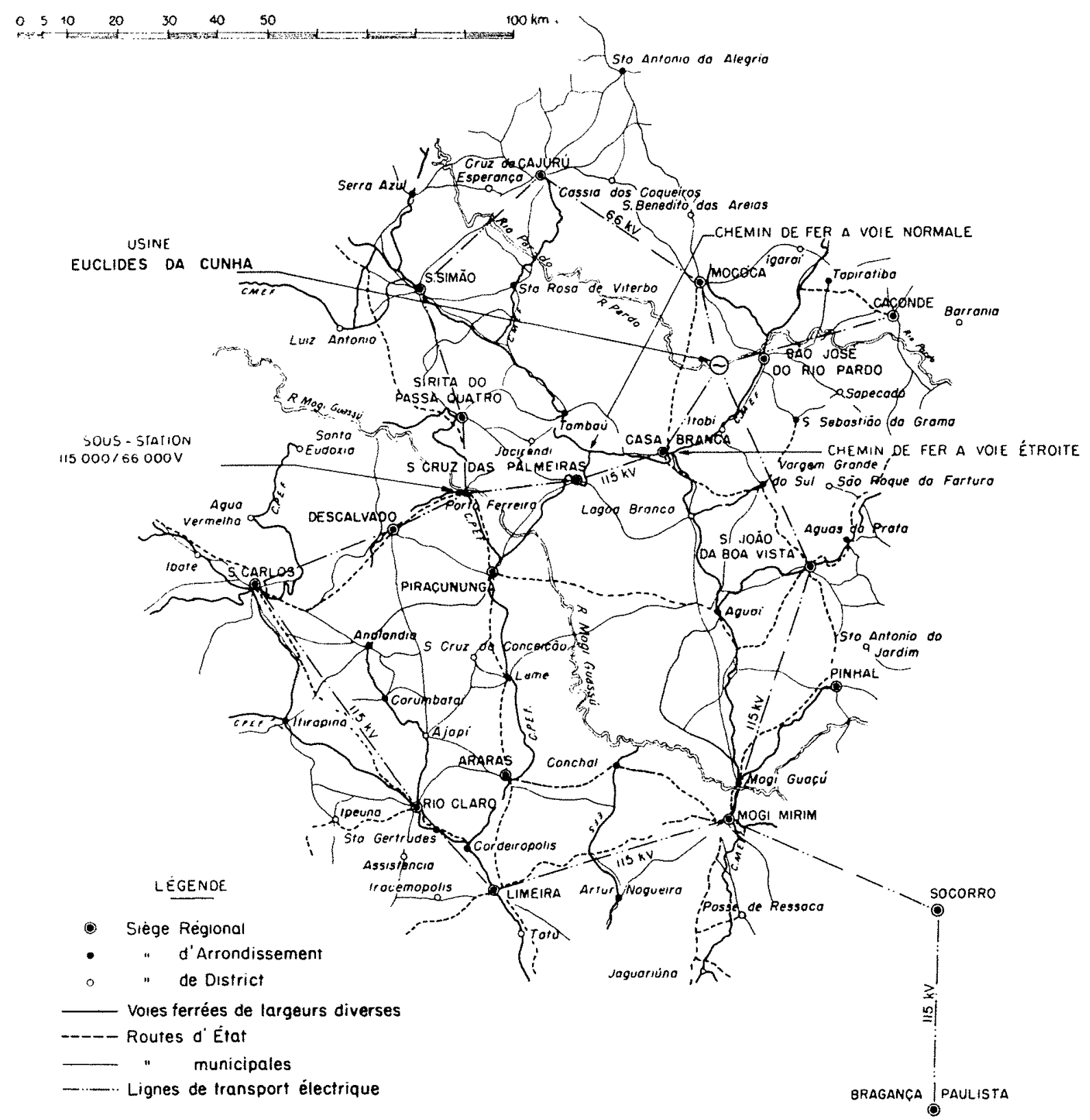

Fig. 10. - Schéma du réseau des transports des installations du Rio Pardo.

Centrale de Limoeiro

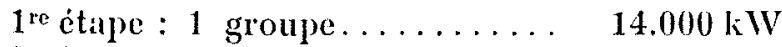

$2^{\circ}$ étape : 1 groupe......... $14.000 \mathrm{~kW}$

$28.000 \mathrm{~kW}$

Cenlrale Euclides da Cunha

$1^{\text {re }}$ étape : 2 groupes. $24.000 \mathrm{~kW} 48.000 \mathrm{~kW}$

$2^{\circ}$ étape : 2 groupes.. $24.000 \mathrm{~kW} \quad 48.000 \mathrm{~kW}$

$96.000 \mathrm{kWV}$

Ces centrales sont situées dans la zone est de l'Etat et sont destinées à fournir leur énergic aux compagnies suivantes :

\section{COMPAGNIES}

1. Cia Paulista de Elelricidade....

2. Cia Paul. de Energia Elétrica...

3. Cia Prada de Eletricidade......

4. Cia Sanjoanense de Eletricidade.

5. S.A. Central El. de Rio Claro....

6. Cia São Simãõ Cajurú.........

7. Cia Luz e Força de Mocóca......

8. Empresa Elétrica Bragsantina....

9. Firme particuliere...........

10. Firme particulière.............

11. Cia Geral de Eletricidade........

12. Cia Sul Mineira de En. Elétrica. .

Total. .
Supericie

2.473

2.322

1.860

1.365

(6. 461

2.763

829

1.052

110

299

5.134

994
I'opulation

66.751

87.371

41.067

59.301

242.517

48.565

31.435

52.177

6.951

5.194

119.769

34.309 


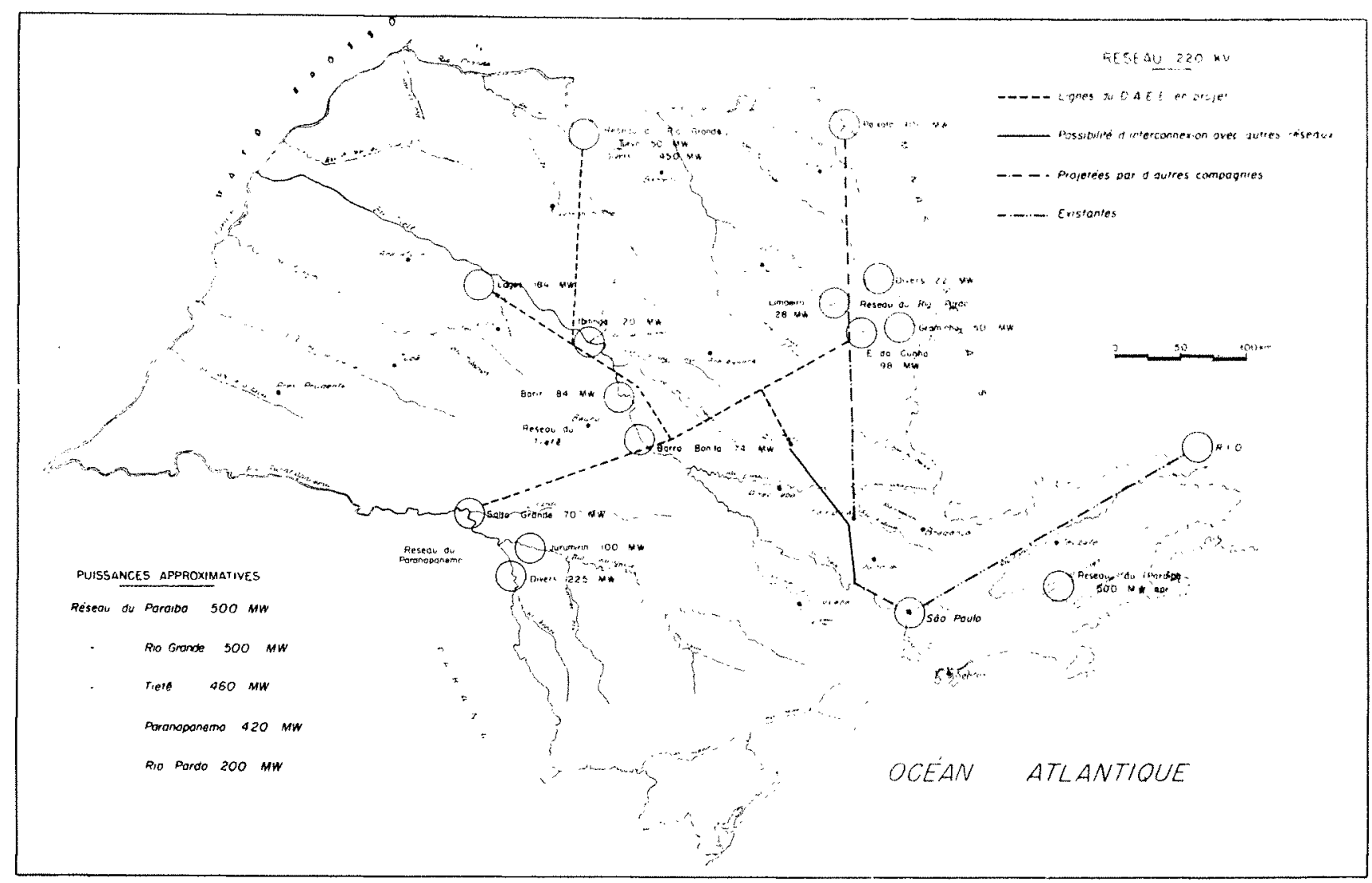

Fí. 11. - Etat de São Paulo : projet pour le transport de force et l'interconnexion

Le graphique de la figure 10 montre le schéma prévu pour les lignes de transmission du système du Rio Pardo.

En complément de ce système, on a étudié la régularisation totale du fleuve, qui s'est avérée possible dans la région de Graminha-Paradouro; le barrage de régularisation générale du Rio Pardo situé dans cette zone est à l'étude; il sera possible d'y installer près de $60.000 \mathrm{~kW}$.

S'intégrant dans le système du Rio Pardo, il faut noter les centrales suivantes, en projet ou en service :

1. Estreito (installée)....... $1.200 \mathrm{~kW}$

2. Vila Biela (à installer)..... $2.400 \mathrm{~kW}$

3. Paradouro (installée)...... $1.200 \mathrm{~kW}$

- Graminha (en étude)...... $60.000 \mathrm{~kW}$

4. Cachoeira do Carmo (en projel).

5. Cachoeira da Bandeira (en projet).

6. Cachocira das Antas (installée) $5.000 \mathrm{~kW}$

Nota : Les centrales Cachoeira do Carmo el Bandeira appartiennent à la Cie Brésilienne d'Aluminium.

\section{VALTKE DU TIETE}

Dans cette région, l'Etat iludie l'utilisation générale du Rio Tietê, dans un triple but :

a) Production d'énergie électrique,

b) Irrigation,

c) Navigation.

Les installations de la Vallée du Tietê sont également à la charge du Département des Eaux et de l'Energic Electrique. Les études relatives a la centrale de Barra Bonita $(75.000 \mathrm{~kW})$ sont en cours, ainsi que les études préliminaires des centrales de Lagos $(120.000 \mathrm{~kW})$ et de lbitinga $(184.000 \mathrm{~kW})$.

\section{Vallike do Paraiba}

Ià, également, le Département procède à l'étude de la régularisation totale du cours supérieur du fleuve où il apparaît très avantageux d'installer la centrale de Caraguatatuba (près de $500.000 \mathrm{~kW}$ ). I'installation de cette centrale est 
basée sur la derivation du Haut-Paraba -..- depuis son point de formation, soit à la jonction de ses affuents Paraitinga el Parabuna jusqu'à la Serra do Mar - la chute obtenue étant de l'ordre de $600 \mathrm{~m}$.

Les eludes faites par le Déparlement démontrent la possibilité de ce détournement sans préjudice pour les installations de Light-Rio de Janeiro, prévues sur ce mème fleuve.

\section{Vathée De: Ribeika}

Dans cette région, le Département étudio la centrale de Capivari (pres de $50.000 \mathrm{~kW}$ ) en ayant pour objectif principal de fournir l'unergie nécessaire à l'électrification du Chemin de Fer de Sorocabana.

\section{PLAN DE L'ETAT CONCERNANT L'INTERCONNEXION DES DIFFÉRENT GROUPES}

D'une façon préliminaire, ef avanl que le projet général d'électrification de l'Etal soit définitivement fixc, lo systime d'interconnexion des divers groupes peut être défini comme suit:

Les syslemes de la Light and Power et de la Cia Paulista de Força e Luz ont déjà établi les tracés généraux de leurs grandes lignes de transport, la Light assurant l'interconnexion São Paulo/Rio grâce à une ligne de $220 \mathrm{kV}$ el $100.000 \mathrm{~kW}$ de puissance; la Paulista de Força
Lu\% a doja clabli les plans d'une ligne principale de $220 \mathrm{kV}$ et de $120.000 \mathrm{~kW}$ de puissance initiale. fui reliera la centrale de Peixolos a la ville de Campinas, son centre consommaleur le plus important.

En récapilulant, nous conshatons que l'Etal éludie ou procède à l'installation des systemes suivants :

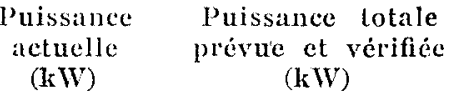

Rio Pardo........ $68.000 \quad 200.000$

Paranapanema .... $60.000 \quad 420.000$

Tietê......... $74.000 \quad \$ 60.000$

Rio Grande (Turvo) . $50.000 \quad 450.000$

Paraiba ........ - $\quad 500.000$

Pour faire face au transport et à l'interconnexion de ces divers systèmes, en ienant compte des systèmes déjà existants, ou cn exécution, de la Light el de la Cia Paulista de Forca e Luz, le Département des Eaux el Energic Electrique a procédè à l'étude de base présentée sur la carte de la figure 11. Ies lignes secondaires et les lignes de distribution seront à 88 et $66 \mathrm{kV}$, avec prédominance de $66 \mathrm{kV}$.

Nous pensons, dans cet exposé, avoir donné un aperecu général de l'évolution de l'énergie ćlectrique dans l'Etat de São Paulo et avoir fait ressortir plus particulièrement la carence actuelle d'énergic et les projets qui ont été élaborés.

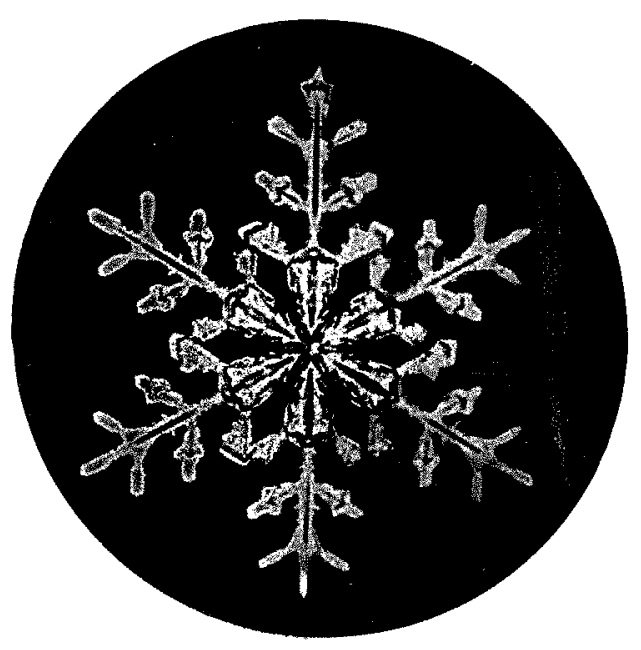

